\title{
Diseño de una empresa de producción de Tilapia en agua dulce en Delicias Chihuahua.
}

Design of a Tilapia production company in freshwater in Delicias, Chihuahua

\author{
Gallegos Carrasco Sarahi Lizzette ${ }^{1,}$ Magaña Magaña José Eduardo ${ }^{2 凶}$, Villarreal \\ Ramírez Víctor Hugo $^{2}$, Kiessling Davison Christian Mauricio ${ }^{2}$, Zúñiga Ávila Gabriel ${ }^{2}$ \\ ${ }^{1}$ Estudiante de la Maestría en Agronegocios, PNPC-CONACYT, ${ }^{2}$ Profesor de \\ la Universidad Autónoma de Chihuahua-Facultad de Ciencias Agrícolas y Forestales, \\ ${ }^{凶}$ Autor para correspondencia: emagana@uach.mx,
}

Recibido: $18 / 02 / 2017$

Aceptado: 30/06/2017

\section{RESUMEN}

La pesca, en agua salada o dulce, es una actividad desde los inicios del ser humano para su alimentación. A la fecha, existe una gran demanda de consumo de pescado fresco, particularmente la Tilapia producida en agua dulce, conociéndose esta actividad como acuicultura. Se analizó la factibilidad técnica, económica y financiera de instalar una empresa de producción de Tilapia en agua dulce en Delicias, Chihuahua con la finalidad de contribuir al desarrollo económico de la región. Se realizaron entrevistas a expertos y a los acuicultores de la región para identificar la especie más adaptable donde resulto ser la Tilapia, considerando el clima y la fuente de agua a utilizar que proviene de pozo profundo. Se analizó el mercado de consumo de pescado fresco en Delicias a través de la aplicación de 276 cuestionarios a manera de entrevista personal. El $68.35 \%$ de los entrevistados tienen familias de 4 a 6 miembros y manifestaron comprar Tilapia. Con un consumo semanal hasta de $2 \mathrm{kgr}$. y destinando cada semana $\$ 160.00$ de su ingreso familiar. El $63.31 \%$ de los encuestados respondieron adquirir el pescado en pescadería el cual lo relacionan con pescado fresco. Los resultados de los análisis económicos y financieros arrojo una TIR de 17\%, una VAN (15\%) \$4,093.85 y B/C (15\%) de 1.0123.que de acuerdo a los criterios de aceptación de cada indicador, se concluye que este proyecto es viable desde el punto de vista técnico, económico, financiero y ambiental.

Palabras Claves: Acuicultura, Tilapia, pescadería, consumo, proyecto de inversión.

\begin{abstract}
ABSTRAC
Fishing, saltwater or freshwater, is an activity since the beginning of human for food being. To date, there is a high demand for fresh fish consumption, particularly Tilapia produced in freshwater, knowing this activity as aquaculture. the technical, economic and financial
\end{abstract}


feasibility of installing a production company Freshwater Tilapia in Delicias, Chihuahua in order to contribute to the economic development of the region was analyzed. interviews with experts and farmers of the region were conducted to identify the most adaptable species which turned out to be the Tilapia, considering the weather and the source of water used that comes from deep well. the consumer market for fresh fish in Delicias through the application of 276 questionnaires by way of personal interview was analyzed. The $68.35 \%$ of respondents have families of four to six members and expressed Tilapia buy. With a weekly consumption up to $2 \mathrm{kgr}$. and allocating each week $\$ 160.00$ of your household income. The $63.31 \%$ of respondents buy the fish in fish which relate it with fresh fish. The results of the economic and financial analysis yielded an IRR of $17 \%$, one NPV (15\%) \$ $4,093.85$ and B / C (15\%) of 1.0123.que according to the acceptance criteria for each indicator, it is concluded that this project it is feasible from the technical, economic, financial and environmental terms.

Keywords: Aquaculture, Tilapia, Fish shop, consumption, investment project.

\section{INTRODUCCION}

Actualmente, la acuicultura va más allá de la cría de peces en estanques o en arroceras. Por razones estadísticas, la Organización de las Naciones Unidas para la Agricultura y la Alimentación (FAO,2003) define la acuicultura como la explotación de organismos acuáticos, incluyendo peces, moluscos, crustáceos y plantas acuáticas. En este caso, explotación implica cierta forma de intervención en el proceso de cría con la fin de mejorar la producción, así como la de asegurar la propiedad de las existencias que están siendo cultivadas

La producción pesquera a nivel mundial ha aumentado en los últimos 50 años y el abastecimiento de peces ha aumentado a una tasa media anual de $3.2 \%$, sobrepasando la tasa de crecimiento poblacional mundial del $1.6 \%$ (FAO, 2014). En los años de 1960 el consumo per cápita mundial de pescado era de 9.9 $\mathrm{kg}$, el cual ha ido aumentando a $19.2 \mathrm{~kg}$ en el 2012 (FAO, 2014) Este considerable aumento se ha dado por el crecimiento demográfico, el aumento de ingresos, la urbanización (FAO, 2014).En México, la acuicultura nace como una actividad complementaria de apoyo social a las comunidades rurales, con lo cual se aumentaría el consumo de proteína animal y mejorar así los niveles nutricionales de la población (Juárez, et al, 1987).

\section{Antecedentes}

En Chihuahua se localizan 26 embalses mayores a 100 has. Así mismo existen 60 mil hectáreas de agua, de estas los 7 principales centros acuícolas son presas mayores de 2,500 a 17, 000 hectáreas de extensión, alrededor de 2 mil obras de almacenamiento como tanques rústicos, presones, bordos ganaderos y pilas de riego, que solo aprovechan para producción pesquera el 10\%, además de 81 granjas acuícolas. Las principales especies cultivadas en el estado de Chihuahua son la trucha, carpa, bagre, mojarra tilapia y lobina y se desarrolla principalmente en la zona serrana de Madera, Guachochi, Bocoyna y Guadalupe y Calvo asi como en la cuenca del Rio Conchos en el Sur.. Se tienen dos principales tipos de producción, 
autoconsumo y la comercial. Existen 9 sociedades cooperativas de producción pesquera las cuales realizan las actividades de captura en los embalses de la Boquilla Municipio de San Francisco de Conchos y Lago Colina, Presa Francisco I Madero en el Municipio de Rosales, Rio Conchos Municipio de Saucillo, Julimes y Presa Luis I León Municipio de Aldama. Actualmente, se cuenta con tres centros acuícolas en la Boquilla, Madera y Guachochi, mismos que tratan de satisfacer los requerimientos de siembra en los estanques. De igual manera existe un centro de recepción en el municipio de San Francisco de Conchos y se cuentan con 268 embarcaciones.

La acuicultura es una de las actividades con mayor potencial y desarrollo en los últimos años en México, la cual produce grandes beneficios sociales y económicos, originando una alimentación con un valor nutricional alto a costos accesibles. Pero no ha sido suficiente (Álvarez, et al, 2012). Es por ello, que la acuicultura constituye una oportunidad de agronegocio en México.

\section{Objetivos}

\section{Objetivo general}

Contribuir al desarrollo económico de la acuicultura en Delicias, Chihuahua.

\section{Objetivos específicos.}

Identificar la especie a producir y su posible adaptación en Delicias, Chihuahua

Analizar el mercado de acuerdo a las variables de consumo y preferencia bajo las condiciones del mercado fresco y congelado.
Formular un proyecto de inversión de acuicultura en Delicias, Chihuahua.

\section{MATERIALES Y METODOS}

\section{Identificación de la especie posible a producirse en Delicias, Chihuahua}

Se analizó cada una de las especies producidas en el estado de Chihuahua, como son: La Tilapia, Trucha, Trucha Arcoíris, Blackbass, Bagre y Carpa. De acuerdo al desarrollo del cultivo, la infraestructura y el agua, el patrón de alimentación de cada una de las especias. Así como la entrevista a expertos de la materia como es un biólogo consultor de los acuicultores de la Región Centro Sur de Chihuahua. Así como la a productores de Tilapia con más de 10 años de experiencia. Concluyendo que la Tilapia es la especie más adaptable a la región.

\section{Localización}

La ubicación geográfica del estudio de caso se concentra en Delicias, localizada en la región Centro Sur del Estado de Chihuahua, colinda al norte con Meoqui al sur y al este con Saucillo, y al oeste con Rosales. Tiene 496 localidades de las cuales 495 son rurales. Importante centro agrícola con 148045 habitantes según el Censo de Población 2015. Se localiza en la latitud norte $28^{\circ} 11^{\prime \prime}$ y longitud oeste $105^{\circ} 28^{\prime \prime}$; a una altitud de 1,170 metros sobre el nivel del mar. Se cuenta con la cercanía del rio San Pedro y de la Presa Francisco I madero. Delicias pertenece al Sistema de Riego 005.

\section{Diseño de la investigación.}

Se analizaron las variables consumo, frecuencia de consumo, sitio de compra, cantidad del ingreso destinado al consumo semanal de pescado, entre otras, mediante un cuestionario con 11 
preguntas directas aplicado en Delicias. Así mismo se elaboró un proyecto de inversión siguiendo la metodología de SAGARPA-FAPPA calculándose indicadores económicos y financieros como la TIR, VAN y Rel B/C. Se utilizó el costo de oportunidad del capital vigente en el mercado de $15 \%$.

Muestreo y tamaño de la muestra

Tamaño de la muestra

Para calcular el tamaño de la muestra se utilizó el intervalo de confianza 95\%

Dónde:

$$
\begin{aligned}
& \mathrm{D}=7 \% \text { de la media } \\
& \bar{x} \quad=0.2548 \\
& \bar{x}=\text { Media estándar, } 3.69
\end{aligned}
$$

El tamaño de la muestra (n) se calculó tomando como base la varianza y la media obtenida de la variable cantidad de consumo semanal del estudio exploratorio. El tamaño final de la muestra al consumidor final fue la siguiente:

$$
\begin{aligned}
& \mathrm{n}=\text { tamaño de la muestra } \\
& \mathrm{Z}^{2}=1.96 \text { con un } 95 \% \\
& \sigma 2=2.12 \\
& \mathrm{D}=7 \% \text { de la } \\
& \text { media } \bar{x}=0.2548 \\
& \bar{x} \text { =Media estándar, } 3.69 \\
& \begin{array}{c}
\mathrm{n}=\left(\underline{1.96)}^{2} \underline{2}_{(0.2548)^{2}}\right. \\
\end{array} \\
& \mathrm{n}=\underline{3.8416 * 4.4944} \\
& 0.0649 \\
& \mathrm{n}=266=267 \text { cuestionarios }
\end{aligned}
$$

Preparación y análisis de datos.

Teniendo los cuestionarios contestados se procedió a la revisión, codificación y verificación de los datos. Procediendo a asignarle los códigos numéricos de literales para representar cada respuesta a cada pregunta. Se utilizó el programa estadístico "Statistica Package forsocial Science (SPSS 15.0)" IBM-SPSS versión 19

\section{RESULTADOS Y DISCUSIONES}

El $68.35 \%$ de los respondentes manifestaron consumir tilapia (Grafica 1), en segundo lugar de preferencia Balck bass y en tercer lugar Carpa.

\section{Grafica 1. Tipo de pescado que consume} usted

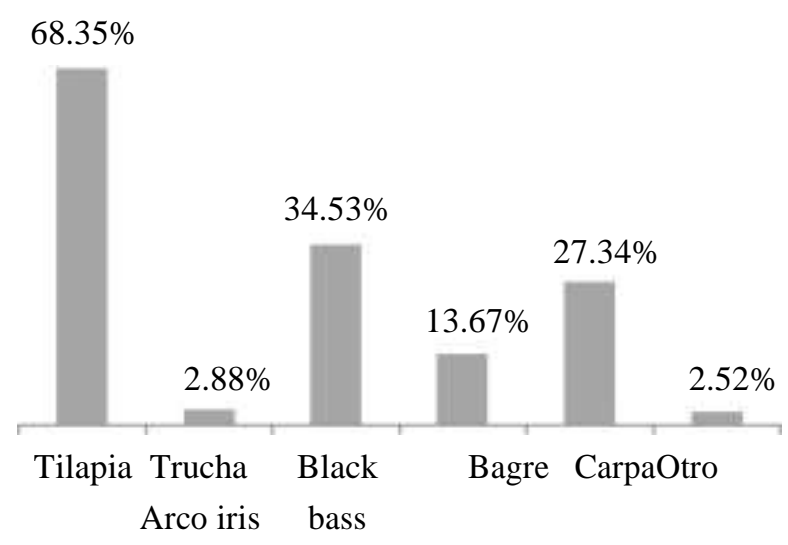

Fuente: Elaboración Propia

Como se observa en la gráfica 2, el 63.31 $\%$ de los consumidores de pescado, adquieren su producto en pescadería,

Gráfica 2. ¿Lugar donde compra el pescado que usted consume?

\section{$63.31 \%$}

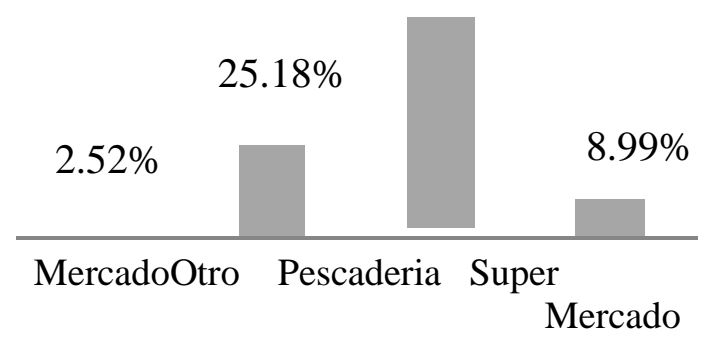

Fuente: Elaboración Propia 
Asociado a la preferencia del sitio de compra en la gráfica 3 se concluye que la frecuencia de visita a la pescadería es semanal con un $36 \%$.

Gráfica 3. Frecuencia de compra en la Pescaderia

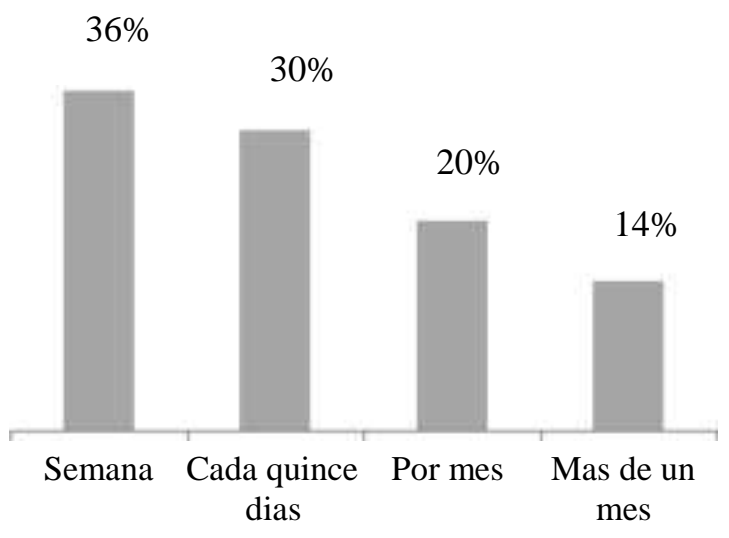

Fuente: Elaboración Propia

En la gráfica 4 se encuentra como resultado que las familias por semana consumen pescado la cantidad de hasta 2 kgr con un $48.56 \%$,

Grafica 4. ¿Cuánto pescado consume
usted y su familia por semana?

$48.56 \%$

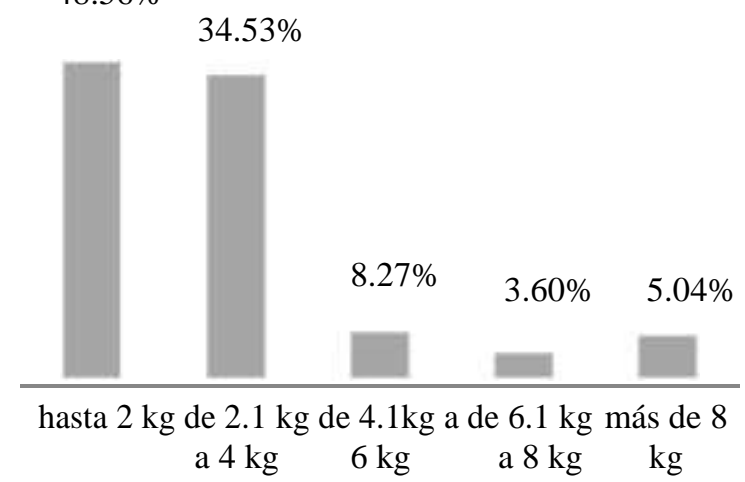

Fuente: Elaboración Propia

Aunado al resultado anterior en la gráfica 5 se observa que en el consumo de hasta
$2 \mathrm{~kg}$ de pescado por familia al mes el en la pescadería es de $56.30 \%$.

Gràfica 5. Lugar de compra para los consumidores de hasta $2 \mathrm{kgr}$.

\section{$56.30 \%$}

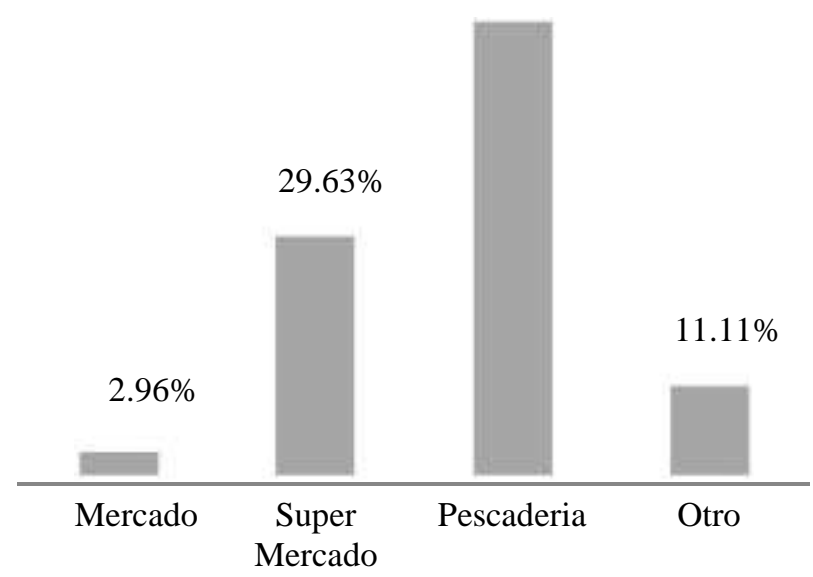

Fuente: Elaboración Propia

Al adquirir el producto en la pescadería el comprador establece preferencia en la higiene del lugar y el empacado con un $36.36 \%$, la frescura del producto con un $34.09 \%$, el aspecto del local con un 27.84 y por ultimo con un porcentaje muy bajo en comparación de los descritos anteriormente con un $1.70 \%$.

Grafica 6. Cuando compra pescado en la percadería ¿en que se fija principalmente?

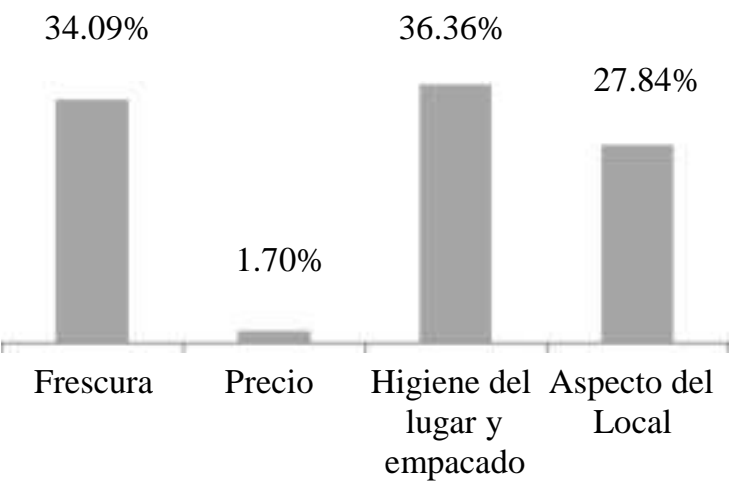

Fuente: Elaboración Propia 
El $39.57 \%$ del consumidor de pescado destina de su ingreso semanal entre $\$ 81$ a $\$ 160$ pesos (grafica 7)

Gráfica 7. Cantidad de ingreso semanal destina alconsumo de pescado

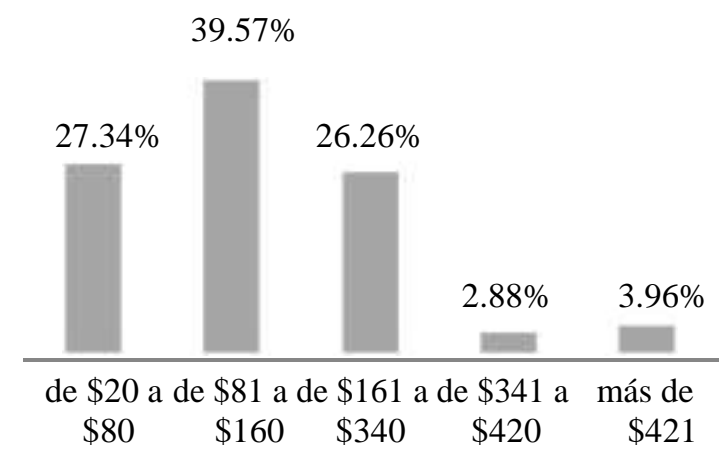

Fuente: Elaboración Propia

En la gráfica 8, nos concluye que las familias consumidora de pescado están compuestas hasta 6 miembros con un $54.68 \%$.

Gráfica 8 ¿Cuántas personas viven en su casa?

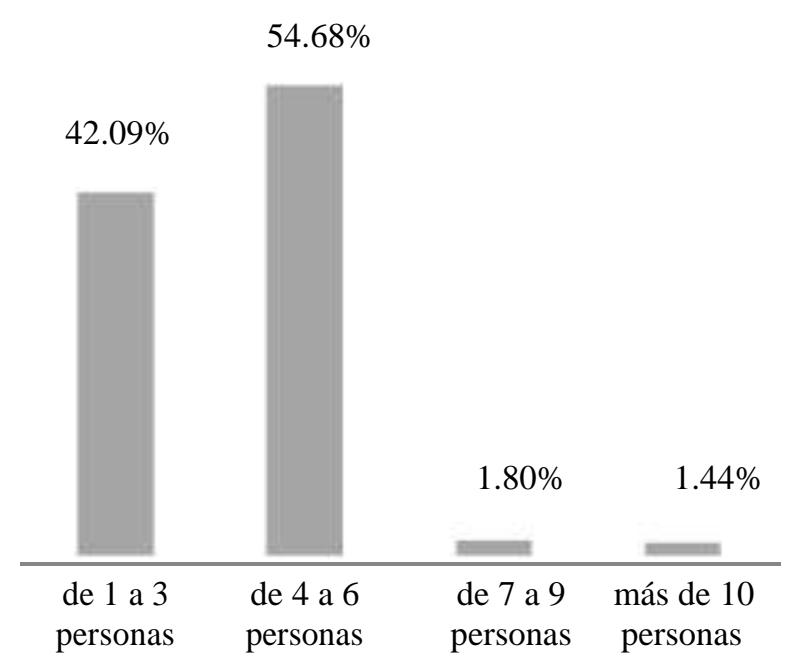

Resultando en la gráfica 9 con un $39.57 \%$ que el ingreso que destinan al consumo de pescado por semana máximo es de $\$ 180.00$.

Grafica 9. Cantidad de ingreso semanal destina al consumo de pescado

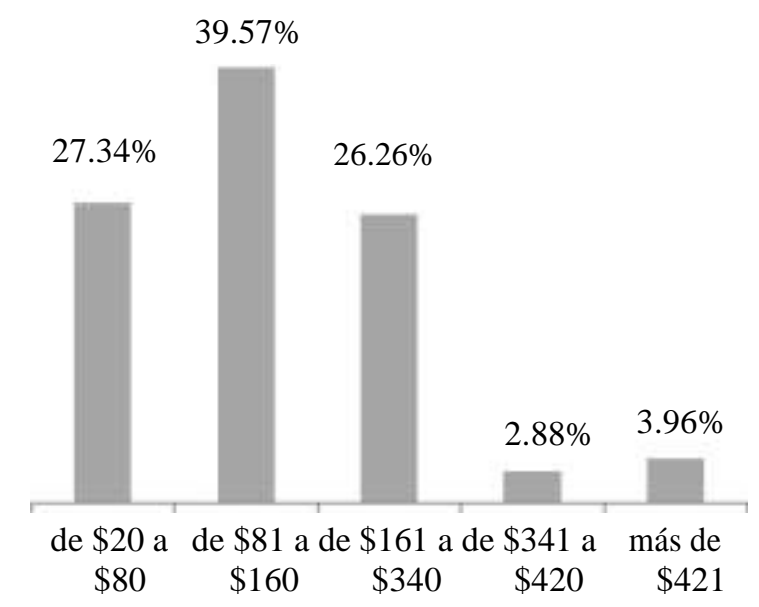

Fuente: Elaboración Propia

El proyecto de inversión presenta indicadores de rentabilidad positivos, lo que significa que es viable y se puede invertir en él, como podemos observar en el (cuadro 4. Indicadores de rentabilidad).

Fuente: Elaboración Propia 
Cuadro 4. Indicadores de rentabilidad

\begin{tabular}{|c|c|c|c|}
\hline $\begin{array}{l}\text { Indicador de } \\
\text { rentabilidad }\end{array}$ & $\begin{array}{l}\text { Porcentaje } \\
\text { /Cantidad }\end{array}$ & Resultado & Interpretación \\
\hline $\begin{array}{l}\text { Tasa interna } \\
\text { de retorno } \\
\text { (TIR) }\end{array}$ & $15 \%$ & $\begin{array}{c}17 \% \\
\text { Se acepta }\end{array}$ & $\begin{array}{l}\text { La TIR es la tasa de interés que paga el proyecto } \\
\text { después de recuperar la inversión inicial. De } \\
\text { acuerdo al criterio de aceptación para proyectos de } \\
\text { desarrollo social que es el de aceptar el proyecto que } \\
\text { tenga una TIR ligeramente mayor que el costo de } \\
\text { oportunidad del capital vigente en el mercado. } \\
\text { Dicho costo de oportunidad fue del } 15 \% \text { y el } \\
\text { proyecto arrojo una TIR del } 17 \% \text {. Bajo esta } \\
\text { condición el presente proyecto es viable. }\end{array}$ \\
\hline $\begin{array}{l}\text { Valor actual } \\
\text { neto (VAN) }\end{array}$ & $15 \%$ & $\$ 4,094$ & $\begin{array}{l}\text { El Valor Actual Neto (VAN) es el valor actual de } \\
\text { los flujos futuros de efectivo descontados a una tasa } \\
\text { del } 15 \% \text {.La VAN del proyecto de inversión } \\
\text { analizado es positivo } \$ 4,094 \text {. (mayor o igual a } 0 \text { ). } \\
\text { Bajo este criterio el proyecto es viable. }\end{array}$ \\
\hline $\begin{array}{c}\text { Relación } \\
\text { beneficio/ } \\
\text { costo (REL } \\
\text { B/C) }\end{array}$ & $15 \%$ & 1.0123 & $\begin{array}{l}\text { Se toman los ingresos y egresos de la proyección } \\
\text { financiera para determinar cuáles son los beneficios } \\
\text { por cada peso que se sacrifica en el proyecto. Se } \\
\text { calculó el proyecto al } 15 \% \text {, el criterio es aceptar el } \\
\text { proyecto cuya relación beneficio /costo sea igual o } \\
\text { mayor a 1. Bajo este criterio la relación } \\
\text { beneficio/costo es de } 1.0123 \text {, por lo tanto se acepta } \\
\text { el proyecto. }\end{array}$ \\
\hline
\end{tabular}




\section{CONCLUSIONES}

En el presente estudio de caso se observó que el $100 \%$ de los entrevistados consumen pescado. De los cuales el $63.31 \%$ prefieren comprarlo fresco en la pescadería. De los tipos de pescado el de mayor demanda con un porcentaje de $68.35 \%$ fue la tilapia. Los resultados de los análisis económicos y financieros nos resultó una TIR de $17 \%$, una VAN $(15 \%) \quad \$ 4,093.85$ y B/C (15\%) de 1.0123.

Por lo tanto se concluye que este proyecto de establecer una empresa de producción de Tilapia en agua dulce en Delicias, Chihuahua es viable.

\section{LITERATURA CITADA}

FAO, 2006, Entendiendo la Acuicultura. Organización de las Naciones Unidas para la Agricultura y la Alimentación.

PROGRAMA RECTOR NACIONAL DE PESCA Y ACUACULTURA. Propuesta para desarrollo del cultivo de tilapia en Mexico.pdf Fecha de consulta 11 Septiembre 2015.

SAGARPA, 2011. GUÍA EMPRESARIAL PARA EL CULTIVO, ENGORDA Y COMERCIALIZACIÓN DE LA TILAPIA (MOJARRA). SAGARPA, CONAPESCA. http://www.fao.org/docrep/field/003/ab46 0s/AB460S05.pdf. Fecha de consulta 10 de septiembre 2015.

http://www.chihuahua.gob.mx/atach2/sdr /canales/Adjuntos/CN_2425CC_3363/ac uacultura.pdf. Fecha de consulta 28 de Agosto 2015.
Revista AquaTIC, no 19, pp. 17-26. Año 2003 http://www.revistaaquatic.com/aquatic/ar t.asp? $\mathrm{t}=\mathrm{p} \& \mathrm{c}=168$ 


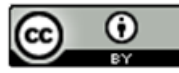

Este tex to está protegido por una licencia licencia Creative Commons $\underline{4.0}$

Usted es libre para Compartir —copiar y redistribuir el material en cualquier medio o form ato-y Adaptar el documento — remezclar, transformar y crear a partir del material- para cualquier propósito, , incluso para fines comerciales, siempre que cumpla la condición de:

Atribución: Usted debe dar crédito a la obra original de manera adecuada, proporcionar un enlace a la licencia, e in dicar si se han realizado cam bios. Puede hacerlo en cualquier form a razonable, pero no de form a tal que sugiera que tiene el apoyo del licenciante o 10 recibe por el uso que hace de la obra.

Resumendelicencia - Textocompletodelalicencia 